\title{
Visualisation and analysis of multi-resolution raster geodata- sets in the cloud
}

\author{
Lassi Lehto *, Jaakko Kähkönen, Juha Oksanen, Tapani Sarjakoski \\ FinnishGeospatial ResearchInstitute, lassi.lehto@nls.fi,jaakko.kahkonen@nls.fi,juha.oksanen@nls.fi, tapani.sarjakoski@nls.fi
}

Keywords: Geodata visualisation, Geodatacube, Multi-resolution, Cloud service

\begin{abstract}
:
Raster geodatasets are an important data source for map-related web applications. However, existing differences in how those datasets are georeferenced, formatted and made available complicate their integration for analysis and visualisation purposes. Furthermore, the inconsistent cell resolutions make it difficult to perform efficient and reliable display of the datasets over a range of scales. In particular, it is challenging to achieve satisfactory user experience in visual exploration of interactive analysis operations carried out on these datasets.

Dataset harmonisation carried out as a pre-processing procedure seems to be necessary for resolving the raster geodata inconsistency challenge. After the pre-processing step, the harmonized datasets have to be stored into an easily accessible data storage, preferably in close connection with the computing platform where the analysis operations are to be performed.
\end{abstract}

Multidimensional datacube has emerged as a conceptual framework for organising a repository of harmonised raster geodatasets. Satellite images have so far been the most popular geospatial application area for datacubes. The set of dimensions of such geodatacube typically consist of two or three coordinate axes, time, and the content theme. While being ingested into the datacube, the involved datasets are brought into a common georeferencing frame, series of cell resolutions, and storage format. The selected harmonised resolution levels can actually be seen as a set of individual geodatacubes.

An initiative has launched in Finland to build a harmonised multi-resolution geodatacube, called GeoCubes Finland, containing some of the most important national geodatasets. The datasets currently incorporated into the geodatacube include digital elevation models together with land cover, superficial deposit, forest inventory and administrative unit datasets. GeoCubes Finland is being built as part of the Finnish Open Geospatial Information Infrastructure for Research (oGIIR) programme, aimed at developing the geospatial research infrastructure of the country. The data storage and the related computing facilities have been implemented on a cloud service platform provided by CSC - IT Center for Science that serves Finnish universities and research institutes.

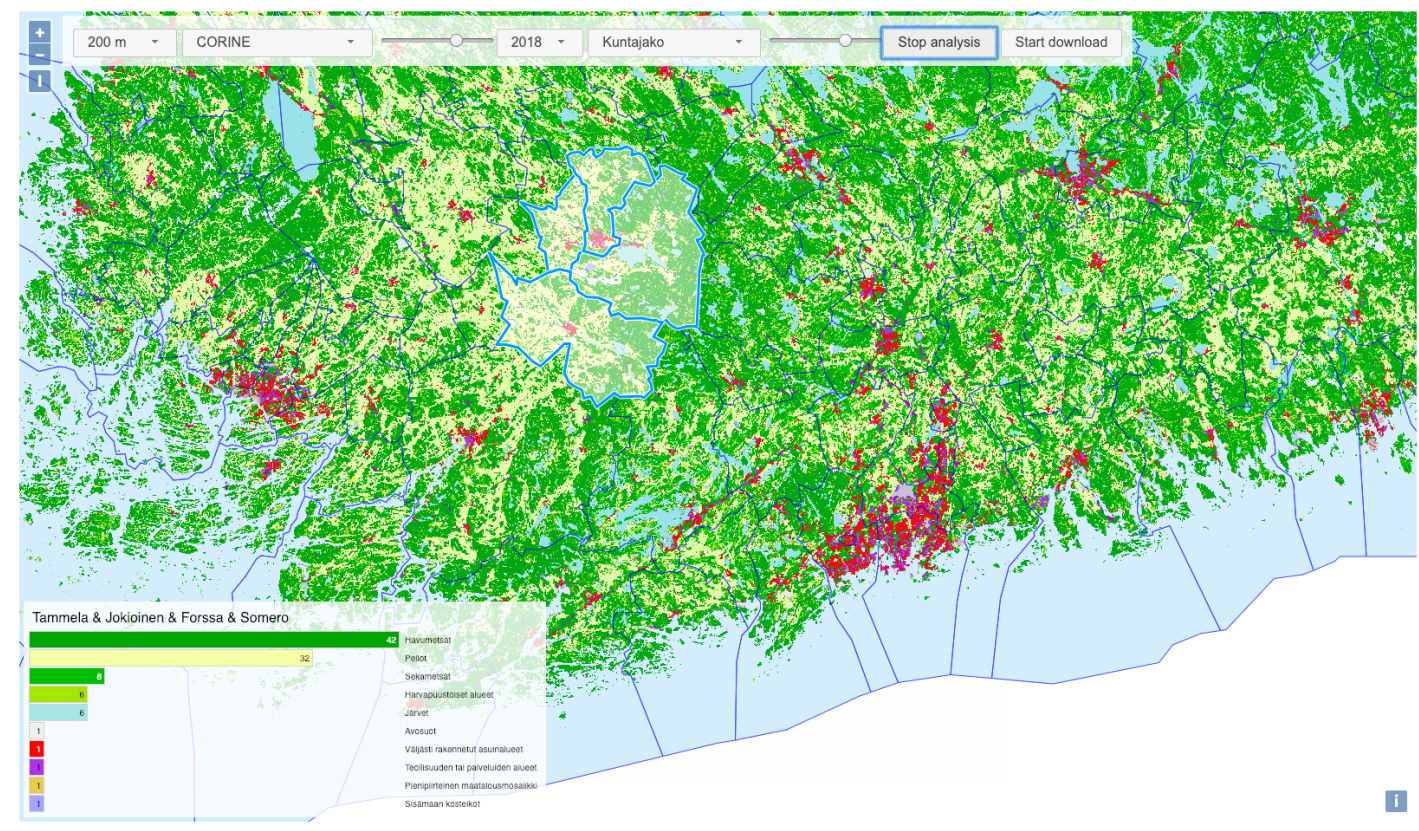

Figure 1. GeoCubes Finland's content layer "CORINE land cover" displayed in a demonstrative web application, together with the analysis results showing the value distribution inside the study area formed by four municipalities. 
GeoCubes Finland has a standardised set of 10 resolution levels going from $1 \mathrm{~m}$ to $1000 \mathrm{~m}$, selected as round values (1, $2,5,10,20,50,100,200,500,1000)$ to support easy integration with other data sources in analysis and visualisation. As the resolution levels are created in a pre-processing step, the generalisation procedures recommended by the responsible data providers can be followed in detail. The applicable maximum resolution level depends on accuracy of each source dataset.

GDAL (Geospatial Data Abstraction Library) is used as the main processing engine while building the GeoCubes Finland data storage. All the data ingestion and provision procedures have been developed in Python language, and essentially depend on GDAL Python API. The geodatacube's raster storage is implemented as cloud-optimized GeoTIFF (COG) files, organised in a tessellation of sixty $100 \mathrm{~km} * 100 \mathrm{~km}$ blocks covering the land area of the country. The virtual format (VRT) mechanism of GDAL is used for combining the individual blocks logically together as a single, continuous coverage.

A set of well-defined and flexible access methods is provided for selecting and retrieving a subset of the geodatacube's contents. The Open Geospatial Consortium's (OGC) standardised Web Coverage Service (WCS) interface can be used for selecting a rectangular area of the indicated content theme from the specified resolution level. For visualisation purposes, also the OGC's Web Map Service (WMS) and Web Map Tile Service (WMTS) interfaces are available.

A flexible custom REST API has been designed to support access, querying and analysis of the geodatacube's contents by theme, resolution level, location and time. In addition to simple selection by a bounding box or block, the available administrative unit layers also facilitate flexible retrieval of content by municipality, county and region of the country. The API also provides access to individual data cell values for interactive exploration of the content layers. In all access operations the user can define, on which resolution level the geodatacube is queried. When downloading data, the user can indicate, whether the result should also include all the coarser levels of resolution.

For demonstration purposes, a few spatial analysis processes have been implemented on the GeoCube Finland's cloud service platform. As the data storage is readily available in the same computing environment, the analysis processes can access the source datasets efficiently. The multi-resolution nature of the geodatacube enables the analysis processes to be run on an appropriate level of accuracy and execution time - depending on the requirements of the task in hand. When the analysis results can be presented in a form of a new map layer, they are readily available for visual exploration over the whole range of scales supported by the geodatacube's resolution levels.

Client environments so far tested with the GeoCubes Finland include QGIS, Jupyter Notebook and two custom-built demonstrative web browser-based applications (Figures 1 and 2). In QGIS the contents of GeoCubes Finland can be accessed via WCS, WMS, WMTS and the REST API interfaces. The VRT mechanism and the cloud-optimised structure of the GeoTIFF images can be utilised for flexible and efficient data transfer. Using these mechanisms, only the actually required cell values, on appropriate resolution levels, are transferred over the network. An interesting web client application also tested with GeoCubes Finland is to perform 3D visualisation of the high-resolution DEM, together with contents of another theme layer - or results of an analysis process - draped on top of it. A demonstrative implementation of this approach has been tested with the three.js library (Figure 2).

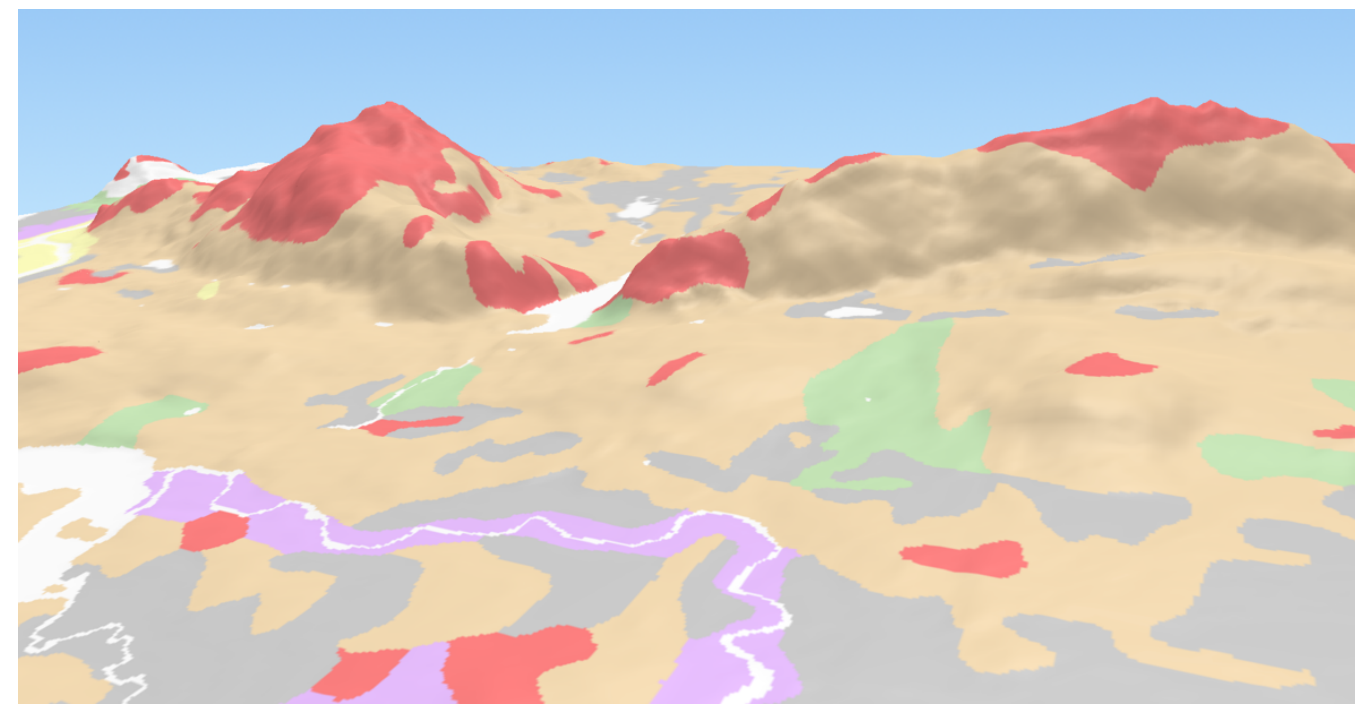

Figure 2. GeoCubes Finland's content layer "Surface Deposits" shown draped over the DEM in a three.js -based client application. 